\title{
A study of extremally disconnected topological spaces
}

\author{
Alexander Arhangel'skii
}

Received: 20 October 2010 / Accepted: 19 January 2011 / Published online: 17 March 2011

(C) The Author(s) 2011. This article is published with open access at SpringerLink.com

\begin{abstract}
We show that if an extremally disconnected space $X$ has a homogeneous compactification, then $X$ is finite. It follows that if a totally bounded topological group has a dense extremally disconnected subspace, then it is finite. The techniques developed in this article also imply that if the square of a topological group $G$ has a dense extremally disconnected subspace, then $G$ is discrete. See also Theorem 3.12. We also establish a sufficient condition for an extremally disconnected topological ring to be discrete (Theorem 3.9). A theorem on the structure of an arbitrary homeomorphism of an extremally disconnected topological group onto itself is proved (see Theorem 3.7 and Corollary 3.8).
\end{abstract}

Keywords Extremally disconnected - Compactification - Topological group . Dyadic compactum $\cdot$ Homogeneous space $\cdot$ Totally bounded group

Mathematics Subject Classification (2000) Primary 54A25; Secondary 54B05

\section{Introduction}

In this article, "a space" is "a Tychonoff topological space". We consider various aspects of the theory of extremally disconnected spaces. A space $X$ is extremally disconnected if the closure of every open subset of $X$ is open (see $[10,12,14]$ ). Obviously,

Communicated by S.K. Jain.

A. Arhangel'skii ( $\varangle)$

Ohio University, Athens, OH 45701, USA

e-mail: arhangel.alex@gmail.com

A. Arhangel'skii

King Abdulaziz University, Jeddah, Saudi Arabia 
every discrete space is extremally disconnected, but the converse is not true. However, every metrizable extremally disconnected space is discrete. Thus, extremal disconnectedness can be considered as a non-trivial generalization of discreteness. On the other hand, it can be also viewed as a very strong case of total disconnectedness and zero-dimensionality, since every extremally disconnected space, obviously, has a base consisting of sets that are both open and closed. Extremally disconnected spaces may seem to be somewhat exotic objects, but Gleason [10], Ponomarev [14], and several other mathematicians have shown that they play a central role in the theory of continuous mappings of compact spaces and, more generally, in the theory of irreducible perfect mappings.

We study extremally disconnected spaces from various points of view. In particular, we consider compactifications of extremally disconnected spaces, we investigate when a space has a dense extremally disconnected subspace, we study how extremal disconnectedness interacts with homogeneity. We also study the structure of homeomorphisms of extremally disconnected spaces and obtain some new results on extremally disconnected topological groups. It is an old problem, more than 40 years old, posed in [1] whether every extremally disconnected topological group is discrete. The negative answer is consistent with $Z F C$ [16], but nobody was able to construct the required example in $Z F C$ only.

Recall that a topological group $G$ is totally bounded, or precompact, if for each open neighbourhood $V$ of the neutral element $e$ some finite collection of the sets of the form $x V$, where $x \in G$, covers $G$. A dyadic compactum is a compact space which can be represented as an image of a topological product of some family of metrizable compacta under a continuous mapping. A standard reference for topological groups is [4]. For definitions in General Topology, see [3] and [7].

\section{Extremally disconnected subspaces, homogeneous extensions, and topological groups}

In this section we consider a general problem: when a topological space $X$ may have a dense extremally disconnected subspace? Notice that any infinite countable metrizable compact space $X$ has a dense extremally disconnected subspace $Y$ consisting of all isolated points of $X$, while any such space $X$ is not extremally disconnected. Thus, a space needn't be extremally disconnected if it has a dense extremally disconnected subspace.

Recall that a space $X$ is homogeneous if for any $x, y \in X$ there exists a homeomorphism $h$ of $X$ onto itself such that $h(x)=y$.

It is convenient to distinguish the following point-wise version of extremal disconnectedness. A point $a$ of a space $X$ is a point of extremal disconnectedness of $X$ if there are no disjoint open sets $U$ and $V$ such that $a$ belongs to the closure of each of them. The next easy to prove statement is a part of the folklore. See, for example [4].

Proposition 2.1 A space $X$ is extremally disconnected if and only if every point of $X$ is a point of extremal disconnectedness of $X$. 
By the way, it follows that if a space is locally extremally disconnected, then it is extremally disconnected.

Here is another simple fact which turns out to be quite useful:

Proposition 2.2 If $Y$ is a dense subspace of $X$, and $a \in Y$, then a is a point of extremal disconnectedness of $Y$ if and only if $a$ is a point of extremal disconnectedness of $X$.

The next statement, easy to prove with the help of the above facts, plays a key role in many arguments below.

Theorem 2.3 If a homogeneous space $X$ contains a dense extremally disconnected subspace $Y$, then $X$ is extremally disconnected.

Proof It follows from Proposition 2.2 that $X$ is extremally disconnected at a dense set of points. Since $X$ is homogeneous, we conclude that $X$ is extremally disconnected at every point. Hence, $X$ is extremally disconnected by Proposition 2.1.

Corollary 2.4 If a semi-topological group $G$ contains a dense extremally disconnected subspace, then $G$ is extremally disconnected.

Proof Indeed, every semi-topological group is a homogeneous space.

Now we can easily prove the following basic fact:

Theorem 2.5 A topological group $G$ is extremally disconnected if and only if its Raikov completion $\rho G$ is extremally disconnected.

Proof Indeed, $\rho G$ is a topological group containing $G$ as a dense subgroup [4]. Therefore, we can apply Corollary 2.4. To establish the opposite implication, it suffices to refer to the fact that every dense subspace of an extremally disconnected space is, obviously, extremally disconnected.

Our next result improves a theorem of Frolik saying that every homogeneous extremally disconnected compact space is finite (see [2,9]).

Theorem 2.6 If a homogeneous compactum X contains a dense extremally disconnected subspace, then $X$ is finite.

Proof It follows from Theorem 2.3 that $X$ is extremally disconnected. It remains to apply the result of Frolik mentioned above.

Corollary 2.7 If $X$ is an infinite extremally disconnected space, then no compactification of $X$ is homogeneous.

Problem 2.8 Does there exist a homogeneous compact space $X$ that can be mapped by a continuous mapping onto some infinite extremally disconnected compact space $Y$ ?

I believe, the answer to the last question is "no". If this is the case, that would be a very strong generalization of Frolik's Theorem.

Since every topological group is a homogeneous space, we immediately obtain from Theorem 2.6 the next corollary: if a compact topological group $G$ has a dense extremally disconnected subspace, then $G$ is finite. We will see below that the last statement can be considerably improved in several directions. 
Theorem 2.9 Every totally bounded topological group $G$ with a dense extremally disconnected subspace is discrete and hence, is finite.

Proof It is well known that the Raikov completion $\rho G$ of any totally bounded topological group $G$ is a compact topological group containing $G$ as a dense topological subgroup (see [4]). Fix a dense extremally disconnected subspace $Y$ of $G$. Then $Y$ is also a dense subspace of $\rho G$. Now we can apply the above observation and conclude that $\rho G$ and $G$ are finite.

The last result improves Corollary 3.7.29 from [4] saying that every totally bounded extremally disconnected topological group is finite. Besides, the proof of Theorem 2.9 given above is much simpler than the proof of Corollary 3.7.29 in [4] based on quite different ideas. Recall that a subset $A$ of a uniform space $X$ is precompact if the closure of $A$ in the uniform completion of $X$ is compact [7]. Every topological group $G$ has a natural two-sided uniformity; the completion of $G$ with respect to this uniformity is the Raikov completion $\rho G$ of $G$ [4].

We can present now an alternative proof of Theore 3.7.28 from [4], on which the proof of Corollary 3.7.29 is based, and to generalize this theorem as follows:

Theorem 2.10 Suppose that $G$ is a topological group with a dense extremally disconnected subspace. Then every precompact subset $A$ of $G$ is finite.

Proof The Raikov completion $\rho G$ is a topological group with a dense extremally disconnected subspace. Therefore, $\rho G$ is extremally disconnected, by Corollary 2.4. Since $\rho G$ is the uniform completion of $G$ with respect to the two-sided uniformity of $G$, and $A$ is precompact, it follows that the closure of $A$ in $\rho G$ is a compact subspace $B$ of $\rho G$. However, every compact subspace of any extremally disconnected topological group is finite, as it was shown in [1]. Hence, the sets $B$ and $A$ are finite.

A compact space $X$ is a dyadic compactum if, for some cardinal number $\tau$, there exists a continuous mapping of the space $D^{\tau}$ onto $X$. Here $D=\{0,1\}$, and $D^{\tau}$ is the topological product of $\tau$ copies of the discrete space $D$.

It is a fundamental theorem of L. Ivanovskij and V. Kuzminov that every compact topological group is a dyadic compactum (for a proof and a reference, see [4]).

A compactification $b X$ of a space $X$ is said to be a dyadic compactification of $X$ if $b X$ is a dyadic compactum.

A space $B$ will be called point-wise sequential if for every non-isolated point $a \in B$ there exists a sequence $\eta=\left\{a_{n}: n \in \omega\right\}$ in $B \backslash\{a\}$ converging to $a$. It is well known that every dyadic compactum is point-wise sequential (see, for example [7]). The next general statement leads to several curious corollaries.

Proposition 2.11 If $Y$ is an extremally disconnected dense subspace of a point-wise sequential space $X$, then $Y$ is discrete.

Proof Assume the contrary, and fix a non-isolated point $a \in Y$. Then $a$ is nonisolated in $X$ as well, and, since $X$ is point-wise sequential, we can fix a sequence $\eta=\left\{b_{n}: n \in \omega\right\}$ in $X$ such that $\eta$ converges to $a$ and $a \neq b_{n} \neq b_{m}$ for any distinct $n, m$ in $\omega$. Now, by a standard inductive argument, we can construct two disjoint open 
sets $U$ and $W$ in $X$ such that $b_{n} \in U$, for each even number $n$, and $b_{n} \in W$, for each odd number $n$. Then $a$ belongs to the intersection of the closures in $Y$ of the open in $Y$ and disjoint sets $U \cap Y$ and $W \cap Y$, which is impossible, since $Y$ is extremally disconnected.

The next result can be compared to results of Efimov in [6].

Corollary 2.12 If a dyadic compactum $B$ has a dense extremally disconnected subspace $Y$, then $Y$ is discrete, countable, and $B$ is metrizable.

Proof Every dyadic compactum is point-wise sequential [7]. Therefore, by Proposition 2.11, $Y$ is discrete. But every dyadic compactum with a dense metrizable subspace is separable and metrizable, according to a Theorem of Efimov [6]. Hence, $B$ is metrizable, and $Y$ is countable.

By Corollary 2.12, the Tychonoff cube $I^{\tau}$, for $\tau>0$, does not contain an extremally disconnected dense subspace.

Another large class of spaces without dense extremally disconnected subspaces is described in the next theorem.

Theorem 2.13 Suppose that $X$ is the product space of an infinite family of regular non-trivial spaces. Then $X$ cannot contain a dense extremally disconnected subspace.

Proof Assume the contrary. To each point $x$ in $X$ a non-trivial sequence converges. We may assume that $x \in Y$, where $Y$ is an extremally disconnected dense subspace of $X$. Fix a sequence $\eta=\left\{x_{n}: n \in \omega\right\}$ converging to $x$ such that distinct members of $\eta$ are distinct points of $X$. Using regularity of $X$, we can define an open neighbourhood $O\left(x_{n}\right)$ of $x_{n}$ in $X$ such that the family $\xi=\left\{O\left(x_{n}\right): n \in \omega\right\}$ is disjoint. Let $V$ be the union of all $O\left(x_{n}\right)$ where $n$ is odd, and $W$ be the union of all $O\left(x_{n}\right)$ where $n$ is even. Then $V \cap Y$ and $W \cap Y$ are disjoint open sets in $Y$ none of which contains $x$. However, $x \in \overline{V \cap Y} \cap \overline{W \cap Y}$, since $Y$ is dense in $X$. This contradicts extremal disconnectedness of $Y$.

Observe that the class of point-wise sequential spaces is much larger than the class of dyadic compacta. This can be seen from the next obvious general statement:

Proposition 2.14 A non-discrete homogeneous space $X$ is point-wise sequential if and only if $X$ contains a non-trivial convergent sequence.

The next statement immediately follows from Propositions 2.11 and 2.14.

Theorem 2.15 If a homogeneous space X contains a non-trivial convergent sequence, then no dense subspace of $X$ is extremally disconnected.

Now it is time to mention the following delicate question posed by Rudin more than 50 years ago and still open: does every infinite compact homogeneous space contain a non-trivial convergent sequence [15]? We can reformulate this problem as follows:

Problem 2.16 Is every homogeneous compact space point-wise sequential? 


\section{Extremal disconnectedness and continuous mappings}

Lemma 3.1 Suppose that $h: X \rightarrow X$ is a homeomorphism of a space $X$ onto itself, and that $a \in X$ is a fixed point under $h$, i.e. $h(a)=a$. Then exactly one of the following conditions is satisfied:

(1) There exists an open set $U$ such that $U$ and $h(U)$ are disjoint, and $a \in \bar{U} \cap \overline{h(U)}$;

(2) There exists an open neighbourhood $O(a)$ of a such that $h(x)=x$ for each $x \in O(a)$.

Proof Since $h$ is a homeomorphism of $X$ onto itself, and $h(a)=a$, condition (1) is equivalent to the next condition (0):

(0) There exists an open set $U$ such that $U$ and $h(U)$ are disjoint, and $a \in \bar{U}$.

Call a subset $V$ of $X h$-simple if $V$ and $h(V)$ are disjoint. By Zorn's Lemma, there is a maximal open $h$-simple set $W$. If $a \in \bar{W}$, then we are done.

So assume that $a \notin \bar{W}$. Then $a \notin \overline{h(W)}$, as it was already explained. Therefore, we can fix an open neighbourhood $O(a)$ of $a$ such that $O(a) \cap W=\varnothing$ and $O(a) \cap h(W)=\varnothing$. Since $h$ is continuous and $h(a)=a$, there exists an open neighbourhood $O_{1}(a)$ of $a$ such that $O_{1}(a) \subset O(a)$ and $h\left(O_{1}(a)\right) \subset O(a)$.

If $h(x)=x$, for each $x \in O_{1}(a)$, then condition 2) is satisfied, and we are done. It remains to consider the case when $h(b) \neq b$ for some $b \in O_{1}(a)$. Then $b \in O(a)$ and $h(b) \in O(a)$; since $h$ is continuous, it follows that we can find an open neighbourhood $W_{1}$ of $b$ such that $W_{1} \subset O(a), h\left(W_{1}\right) \subset O(a)$, and $W_{1} \cap h\left(W_{1}\right)=\varnothing$.

Put $U=W \cup W_{1}$. Clearly, $U$ is open, $U$ and $h(U)$ are disjoint, and $W \subset U$, $W \neq U$. This contradicts maximality of $W$ in the family of all open subsets $V$ of $X$ such that $V$ and $h(V)$ are disjoint.

The next result belongs to Frolik [8].

Corollary 3.2 If $h$ is a homeomorphism of an extremally disconnected space $X$ onto itself, and $x$ is a point of $X$ such that $h(x)=x$, then there exists an open neighbourhood $U$ of $x$ such that $h(y)=y$ for each $y \in U$.

Proof This statement follows from Lemma 3.1. Indeed, condition (1) cannot hold, since $X$ is extremally disconnected.

Our approach permits to generalize Corollary 3.2 as follows:

Corollary 3.3 Suppose that $h$ is a homeomorphism of a space $X$ onto itself, and $x$ is a point of $X$ such that $h(x)=x$. Suppose further that $x$ is a point of extremal disconnectedness of $X$. Then there exists an open neighbourhood $O(x)$ of $x$ such that $h(y)=y$ for each $y \in O(x)$.

The last results can be used to compare homeomorphisms of extremally disconnected spaces. Indeed, we have:

Theorem 3.4 Suppose that $f$ and $g$ are homeomorphisms of a space X onto itself, and that $x$ is a point of extremal disconnectedness of $X$ such that $f(x)=g(x)$. Then there exists an open neighbourhood $O(x)$ of $x$ such that $f(y)=g(y)$ for every $y \in O(x)$. 
Proof Put $h=g^{-1} f$. Clearly, $h$ is a homeomorphism of $X$ onto itself, and $h(x)=x$. By Corollary 3.3, there exists an open neighbourhood $O(x)$ of $x$ such that $h(y)=y$ for every $y \in O(x)$. Then $f(y)=g(y)$, for each $y$ in $O(x)$.

Corollary 3.2 can be used to give a simple proof of the next statement:

Theorem 3.5 A topological group $G$ is discrete if and only if the space $G \times G$ is extremally disconnected.

Proof We have to show that if $G \times G$ is extremally disconnected, then the neutral element $e$ of $G$ is isolated in $G$. Assume the contrary. Let $A=\Delta_{G}=\{(x, x): x \in G\}$ be the diagonal of $G \times G$ and $B=\{(x, e): x \in G\}$. Put $h((x, y))=\left(x, x^{-1} y\right)$, for each $(x, y) \in G \times G$. Then, clearly, $h$ is a homeomorphism of $G \times G$ onto itself, and $h((e, e))=(e, e)$. It is also obvious that $h(A)=B$ and $A \cap B=\{(e, e)\}$. It follows that $h(z) \neq z$ for every $z \in A \backslash\{(e, e)\}$. Since the point $(e, e)$ is not isolated in the subspace $A$, it follows that an arbitrary open neighbourhood $O((e, e))$ of $(e, e)$ in $G \times G$ contains a point $z$ such that $h(z) \neq z$. However, this is impossible by Corollary 3.3, since the space $G \times G$ is extremally disconnected, $h$ is a homeomorphism of this space onto itself, and $h((e, e))=(e, e)$.

The proof of Theorem 3.5 shows that the next more general statement holds. For a definition of a rectifiable space see [11].

Theorem 3.6 A rectifiable space $X$ is discrete if and only if the space $X \times X$ is extremally disconnected.

Recall that, consistently, there exists a non-discrete extremally disconnected topological group [16]. On the other hand, there exist extremally disconnected non-discrete semi-topological groups (see [4,5]). These spaces are homogeneous; therefore, there are many homeomorphisms of spaces of this kind onto themselves. However, a theorem below shows that every homeomorphism of an extremally disconnected semi-topological group onto itself has a rather standard structure.

Let $G$ be a left topological group, and $f$ be a homeomorphism of the space $G$ onto itself. We will say that $f$ is locally trivial if for every $a \in G$ there exist $c \in G$ and an open neighbourhood $O(a)$ of $a$ such that $f(x)=c x$ for every $x \in O(a)$. For example, if $G$ is discrete, then every homeomorphism of $G$ onto itself is locally trivial.

For $c \in G$, we denote by $h_{c}$ the left translation by $c$, that is, $h_{c}$ is the homeomorphism of the space $G$ onto itself given by the rule: $h_{c}(x)=c x$, for every $x \in G$. It is obvious and well known that $h_{b}(x) \neq h_{c}(x)$ whenever $b \neq c$ and $x \in G$.

Theorem 3.7 Suppose that $G$ is an extremally disconnected left topological group. Then every homeomorphism $f$ of the space $G$ onto itself is locally trivial.

Proof Take any $a \in G$, and put $b=f(a), c=b a^{-1}$. Then $h_{c}(a)=c a=b=f(a)$. It follows from Theorem 3.4 that there exists an open neighbourhood $O(a)$ of $a$ such that $f(y)=h_{c}(y)=c y$ for every $y \in O(a)$. Thus, $f$ is locally trivial.

Obviously, Theorem 3.7 can be formulated in a slightly stronger way: 
Corollary 3.8 Let $f$ be an arbitrary homeomorphism of an extremally disconnected left topological group $G$ onto itself. Then there exists a disjoint open covering $\gamma$ of the space $G$ such that, for every $U \in \gamma$, the restriction of $f$ to $U$ coincides with the restriction of the left translation $h_{c}$ to $U$, for some $c \in G$.

Proof We use the preceding theorem and, to achieve disjointness of $\gamma$, we refer to the fact that, if $c \neq b$, where $c, b \in G$, then $h_{b}(x) \neq h_{c}(x)$, for any $x \in G$.

Remark If $G$ in the above statement is compact, then the family $\gamma$ in it is finite. If $G$ is Lindelöf or the Souslin number of the space $G$ is countable, then $\gamma$ is countable.

Theorem 3.9 Suppose that $G$ is an extremally disconnected topological ring with the unity 1 and with no non-trivial divisors of 0 . Suppose further that $c$ is a non-zero element of $G$ such that $c \neq 1$ and $c^{-1}$ is defined. Then $G$ is discrete.

Proof Consider the mapping $m_{c}$ of $G$ into itself given by the rule: $m_{c}(x)=c x$, for every $x \in G$. Clearly, $m_{c}$ is continuous and $m_{c}(0)=0$.

Claim 1 The mapping $m_{c}$ is one-to-one.

Indeed, if $m_{c}(a)=m_{c}(b)$, then $c a=c b$. Since $c$ has an inverse, it follows that $a=b$.

Claim 2 The mapping $m_{c}$ is onto, that is, $m_{c}(G)=G$. $b$.

Indeed, take any $b \in G$, and put $a=c^{-1} b$. Then, clearly, $m_{c}(a)=c a=\left(c c^{-1}\right) b=$

It follows from Claims 1 and 2 that the inverse to the mapping $h=m_{c}$ exists. It is also clear that the inverse to $h$ is given by the formula: $h^{-1}(x)=c^{-1} x$, for every $x \in G$. Therefore, $h^{-1}$ is also continuous. Hence, $h$ is a homeomorphism of $G$ onto itself.

Since the space $G$ is extremally disconnected and $h(0)=0$, it follows from Corollary 3.2 that there exists an open neighbourhood $U$ of 0 in $G$ such that $h(x)=x$, for every $x \in U$.

Fix an arbitrary $b \in U$. We have $b=h(b)=c b$. Hence, $c b-1 b=0$. It follows that $(c-1) b=0$. Since $c-1 \neq 0$ and $G$ does not have non-trivial divisors of 0 , we conclude that $b=0$. Thus, $U=\{0\}$, that is, the point 0 is isolated in $G$, and $G$ is discrete.

The above theorem improves the following result from [1] (see also [4]):

Corollary 3.10 Every extremally disconnected topological field is discrete.

Corollary 3.2 implies the following unusual connection between any two topological group operations given on the same extremally disconnected space:

Proposition 3.11 Suppose that on an extremally disconnected space $G$ two group operations are given each of which turns $G$ into a topological group with the same neutral element $e$ and with the topology given on $G$. Then, for every $b \in G$, there exists an open neighbourhood $V$ of e such that the product operations coincide for any $a$ in $V$ whenever the second factor is $b$. 
In connection with Theorems 3.5 and 3.6, we should mention that for every extremally disconnected space which is not necessarily a topological group the following statement holds:

Theorem 3.12 If $X$ and $Y$ are space such that $X \times Y$ is extremally disconnected, then in at least one of the spaces $X$ and $Y$ every countable subset is closed.

Proof We argue by contradiction. Assume that $A=\left\{a_{n}: n \in \omega\right\}$ is a non-closed countable subset of $X$, and fix $b \in \bar{A} \backslash A$. Assume also that $P=\left\{p_{n}: n \in \omega\right\}$ is a non-closed countable subset of $Y$, and fix $q \in \bar{P} \backslash P$.

Put $c_{n}=\left(b, a_{n}\right), d_{n}=\left(p_{n}, q\right), C=\left\{c_{n}: n \in \omega\right\}, D=\left\{d_{n}: n \in \omega\right\}$, and $s=(b, q)$. Clearly, $\bar{C} \cap \bar{D}=\{s\}$.

Since $C$ and $D$ are countable, and $s$ is not in $C \cup D$, we can easily construct, by induction, open sets $V_{n}$ and $W_{n}$ in $X \times Y$ for $n \in \omega$ such that $V_{i} \subset V_{n}, W_{i} \subset W_{n}$, $c_{n} \in V_{n}, d_{n} \in W_{n}, s \notin V_{n} \cup W_{n}$, and $V_{n} \cap W_{n}=\varnothing$, whenever $i, n \in \omega$ are such that $i \leq n$.

Put $V=\cup\left\{V_{n}: n \in \omega\right\}$ and $W=\cup\left\{W_{n}: n \in \omega\right\}$. The sets $V$ and $W$ are open and disjoint, $C \subset V$, and $D \subset W$. It follows that $\bar{V} \cap W=\varnothing$ and $s \in \bar{V} \cap \bar{W}$. Hence, the closure of $V$ is not open, and the space $X \times Y$ is not extremally disconnected. This contradiction completes the proof.

In connection with the above theorem and its corollaries below, see [13], where, in particular, some related consistency results are established.

Corollary 3.13 If $X$ and $Y$ are separable spaces such that $X \times Y$ is extremally disconnected, then at least one of the spaces $X$ and $Y$ is discrete.

Corollary 3.14 If $X$ and $Y$ are spaces of countable tightness such that $X \times Y$ is extremally disconnected, then at least one of the spaces $X$ and $Y$ is discrete.

Notice that Sirota's extremally disconnected non-discrete topological group constructed under $\mathrm{CH}$ in [16] is countable. Hence, its tightness is countable, and we cannot weaken the assumption in the above statement that $X \times Y$ is extremally disconnected to the assumption that $X$ is extremally disconnected.

Corollary 3.15 If $X$ and $Y$ are countably compact spaces such that $X \times Y$ is extremally disconnected, then at least one of the spaces $X$ and $Y$ is discrete.

Open Access This article is distributed under the terms of the Creative Commons Attribution License which permits any use, distribution and reproduction in any medium, provided the original author(s) and source are credited.

\section{References}

1. Arhangel'skii, A.V.: Groupes topologiques extremalement discontinus. C. R. Acad. Sci. Paris 265, 822-825 (1967)

2. Arhangel'skii, A.V.: The structure and classification of topological spaces and cardinal invariants. Russ. Math. Surv. 33, 33-96 (1978) 
3. Arhangel'skii, A.V., Ponomarev, V.I.: Fundamentals of general topology in problems and exercises, Izdat. "Nauka”, Moskva, 1974, (in Russian) (English translation: ser. Mathematics and its Applications, D. Reidel Publishing Co., Dordrecht-Boston, Mass., 1984. xvi+415 pp. Polish translation: Panstwowe Wydawnictwo Naukowe (PWN), Warsaw, 1986. 456 pp)

4. Arhangel'skii, A.V., Tkachenko, M.G.: Topological groups and related structures. Atlantis Press, Amsterdam (2008)

5. Efimov, B.A.: Absolutes of homogeneous spaces. Dokl. AN SSSR 179(2), 271-274 (1968)

6. Efimov, B.A.: Mappings and embeddings of dyadic spaces. Matem. Sb. 103, 1 (1977)

7. Engelking, R.: General Topology. PWN, Warszawa (1977)

8. Frolik, Z.: Homogeneity problems for extremally disconnected spaces. Comment. Math. Univ. Carolin. 8, 757-763 (1967)

9. Frolik, Z.: Fixed points of maps of extremally disconnected spaces and complete Boolean Algebras. Bull. Acad. Polon. Sci. Ser. Math. Astronom. Phys. 16, 269-275 (1968)

10. Gleason, A.M.: Projective topological spaces. Ill. J. Math. 2(4), 482-489 (1988)

11. Gul'ko, A.S.: Rectifiable spaces. Topol. Appl. 68, 107-112 (1996)

12. Iliadis, S.D.: Absolutes of Hausdorff spaces. Dokl. AN SSSR 149, 22-25 (1963)

13. Malykhin, V.I.: Products of extremally disconnected spaces and measurable cardinals. In: Proceedings of the Third Tiraspol Symposium, pp. 67-69 (1973)

14. Ponomarev, V.I.: On the absolute of a topological space. Dokl. AN SSSR 149, 26-29 (1963)

15. Rudin, W.: Homogeneity problems in the theory of Čech compactifications. Duke Math. J. 23, 409-419 (1956)

16. Sirota, S.: A product of topological groups and extremal disconnectedness. Matem. Sb. (NS) 79(121), 179-192 (1969) (in Russian) 